\title{
ACQUISITION OF EMG SIGNAL USING PIC18F87K22 MICROCONTROLLER
}

\author{
Nemanja Peruničić, \\ Đorđe Novaković
}

Faculty of Technical Sciences, University of Novi Sad, Novi Sad, Serbia
Correspondence:

Đorđe Novaković

e-mail:

djordjenovakovic@uns.ac.rs

\begin{abstract}
:
Purpose of this paper is to give an insight into the basics of gathering and processing of bio-potentials of our muscles. First, we address the laws and principles on which process of measuring is based upon; namely we examine what is measured, and how? Afterwards there is a detailed description of the measuring system - in our case it is a PIC18F87K22 microcontroller based system with a specially designed click-board on it. Firmware which controls the system behavior is written in mikroC development environment. Finally, the measurement results are acquainted in the $\log$ format and presented by MATLAB program.
\end{abstract}

Keywords:

EMG, biceps, PIC, UART, mikroC.

\section{INTRODUCTION}

Information from our environment is gathered by sensory receptors, who process them to the brain. Brain sends a response, put into action by actuators (smooth and skeletal muscles). Nerves represent pathways that connect mentioned entireties; impulses that cause depolarization of muscle cells are transmitted along them. Depolarization occurs when an outside energy causes the regrouping of negative and positive ions, in and out of a muscle cell, via electrical gradient (and supported by diffusion gradient) - this causes chemical reactions, which in turn cause mechanical work. Works of many cells are combined into muscle contraction.

Intensity of contraction is proportional to intensity of the measured signal, and it depends on the number of impulses brought by the nerves. Empirical connection between the frequency of impulses $f(t)$ (given in pps - pulse per second) and intensity of contraction $\mathrm{p}(\mathrm{t})$ (non-dimensional) is

$$
p(t)=e^{(f(t)-B) / A}
$$

And the model for EMG signal is given from [1]

$$
e(n)=w(n)+\sum_{r=1}^{N} p(r) \times h(n-r)
$$


Where $\mathrm{h}(\mathrm{n}-\mathrm{r})$ is screening function; it's used to separate individual components from the intensity, and $\mathrm{w}(\mathrm{n})$ is additive white Gaussian noise. Digital electronic circuits are used for signal analysis so discrete time is introduced. Intensity $\mathrm{p}(\mathrm{n})$ is actually a result of superposition of impulses from individual motor units (group of muscle cells innervated by single nerve), that number $\mathrm{N}+\mathrm{M}$ inside of a muscle, and differ in number and width of its cells. Not all motor units will become activated with arbitrary contraction $(\mathrm{M})$, because of their different thresholds.

Although the number of pps can be measured with electroneurography device, and models exist for w(n), it is impossible to calculate A and B with great accuracy. Therefore $e(t)$ is measured directly.

\section{BASICS OF ELECTROMYOGRAPHY}

Electromyography (EMG) is a method for measuring electrical activity of skeletal muscles for diagnostic purposes, or monitoring during therapy for inflammations, injuries and muscle atrophy. It can be used for clinical purposes or scientific research; where the first one will, generally, have higher standards. EMG is usually non-invasive, but it can be - if needle-electrodes are used. Stimulating electrodes can be used in a combination with measuring ones, for testing nerve conductivity.

\section{Measurment ranges}

The muscles will generate different signals, plus we have the obvious differences between individuals - but, as far as humans go EMG frequency range is from 10 to $10000 \mathrm{~Hz}$, and amplitude from 0.05 to $5 \mathrm{mV}$.

In this paper we are considering the use of scientific research non-invasive EMG, set to give valid results for signals up to $500 \mathrm{~Hz}$ from left biceps. Frequency limitation isn't a problem since higher frequency components sometime don't exist, or are negligible.

\section{Surface measuring electrodes}

Electrodes for gathering physiological signals (EMG, amongst others) are meant for multiple usage, and made from silver of great purity coated with a thin layer of silver-chloride $(\mathrm{Ag} / \mathrm{AgCl})$. They are shaped like a disk, $10 \mathrm{~mm}$ diameter, and are located inside a plastic isolation package. Each electrode actually consists of two parts, meant to be joint like a button. Part that lays in is connected to a self-adhesive pillow. One-channel bipolar measurement is used, i.e. we are measuring the voltage between the pair of active electrodes, whilst the third one (called Driven Right Leg) is positioned anywhere on the body. It doesn't matter which one is used as DRL, they are identical. Everything given about the electrodes is according to [2]. Fig. 1 gives an example of the electrode placement.

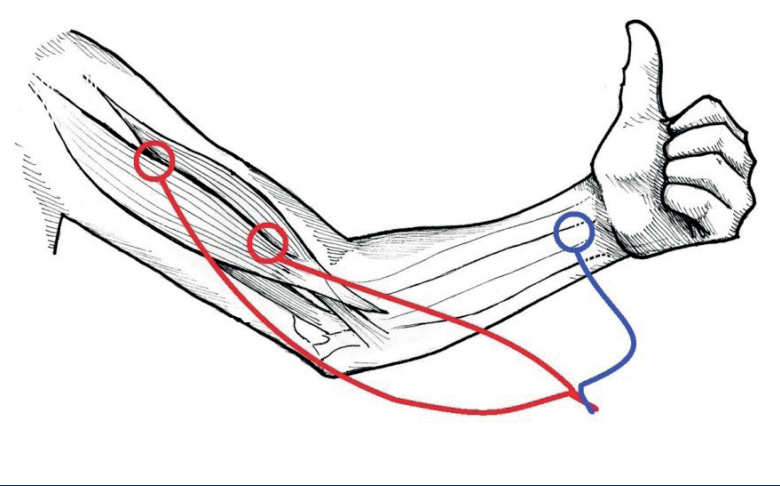

Fig. 1. Electrode placement

Components of EMG system

Signal from the electrodes must be amplified at least 10000 times, plus we have to worry about unwanted signals coming from other parts of the body, or from external electro-magnetic fields. High-pass filter is used for eliminating unwanted signals, and low-pass filter for movement artifacts and offsets from the amplifier. DRL electrode eliminates common voltage from instrumentation amplifier. Parasitic capacitance and inductance are eliminated with twisted and armored cables, respectively.

Since it is commonly used with other devices, ground loop can become a problem. Protection circuit protects both patient and the device from high voltages and electrostatic discharge. All technical solutions mentioned above are found in [3]. Fig. 2 shows the elements that every EMG must have.

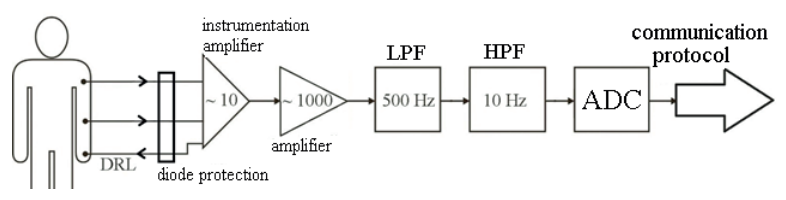

Fig. 2. Principal block scheme 


\section{MICROCONTROLLER MODULE}

Microcontroller is a digitally integrated electronic circuit for independent real-time control of devices and processes, comprising: microprocessor, memory, digital and analog inputs / outputs, oscillators, timers, counters, converters, comparators, communication interfaces, etc. The chip itself is embedded on a development board, rich in various indicators and connectors, with multiple applications.

All microcontrollers are distinguished by the use of interrupt routines - interrupt, which begins when the pre-set conditions are met (the program constantly checks them), and the operations that were executed until that moment are put on hold. Programmable Intelligent Computer (PIC) is one of the types of microcontrollers; division is done according to architecture, method of instruction and application. Click-boards are specially designed integrated circuits designed for a specific job, which connect to a microcontroller through a 16-pin set - mikroBUS socket. The PIC18F87K22 microcontroller, together with the click-board EMG click, plays the role of an EMG device.

The Central Processing Unit (CPU; microprocessor) is the main part of any microcontroller. His role is to receive instructions, process them and hire other units as needed. It consists of

- Instruction decoder - converts binary code into program commands.

- Arithmetic-logical unit (ALU) - performs mathematical and logical operations over data.

- Accumulator - register containing the data that ALU is currently working on.

- Status register - contains additional information about the numbers in the accumulator (e.g. are they positive).

Storage is shared depending on what we store

- Read Only Memory (ROM) - for the code, the size of several tens of $\mathrm{kB}$. In particular, Flash ROM with floating gate transistors is used, which can keep logical state without power supply.

- Random Access Memory (RAM) - for data; whether it's the end results or in-between, the size of a few $\mathrm{kB}$. It's made of general purpose registers (for data values and addresses) and special registers (such as status register).

Input and output pins are registers that connect the microcontroller to the environment. Whether it will be analogue or digital is predefined in their purpose, and the user determines who will receive or send signals (information about this is recorded in the TRIS special register). Oscillator is a piezoelectric crystal with a resonant frequency set (in our case $16 \mathrm{MHz}$ ), which gives a working clock to the rest of the circuit. The timer counts the impulses from the oscillator, but since each instruction needs four oscillations, the timer is incremented once for every $0.25 \mu$ s (reciprocal value of $1 / 4$ of $16 \mathrm{MHz}$ ). When the timer's registry is full, it comes to an interrupt - and some time has passed. The counter counts the impulses of the input signals (e.g. objects on the production line, detected with industrial sensors).

\section{Connecting the elements}

It is clear that there must be a system of communication between parts of the microcontroller. PICs have a Harvard architecture, which uses two physically separate buses; for data transmission and memory addresses where they are located, or need to be. Bus can be of different size, and we can perform parallel operations, speeding up the work of the entire system. Fig. 4 gives an example of communication.

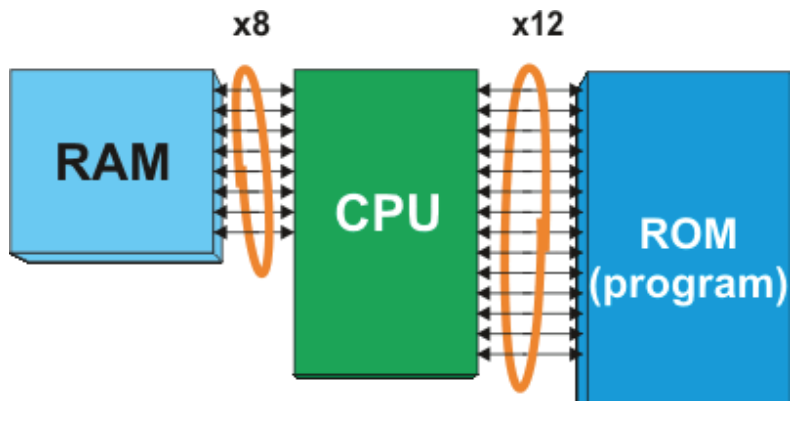

Fig. 3. Harvard architecture

Through the highway, the data is sent by bit; starting from e.g. the one with the highest weight factor, i.e. information is sent in full through one bus. We call this approach Serial Communication, but it is further divided according to the number of connections between the two elements. The best-known example is the Universal Serial Bus (USB), through which computer and microcontroller share information. For the communication between microcontroller and EMG click Universal Asynchronous Receiver-Transmitter (UART) protocol is used. Asynchronous means that the clock is not given 
by the synchronization oscillator, but the transmitter and receiver have a predefined common speed (expressed in bps - bits per second). The morphology of the UART code is given in Fig. 5.

- Start bit; always 0 - tells the receiver to turn on its own internal clock; to go and follow what comes to him.

- Data is often sent in 8-bit format; LSB comes first.

- The parity bit can be added as a check of the correct reading of the receiver. It has two modes: even parity and odd parity - depending on whether we want a even or an odd number of counted logical ones 1 (in the code word in front of it) to give logic 1 in parity.

- Stop bit; always 1 - there can be more than one.

With the microcontroller used here, the maximum transmission speed is $10^{6} \mathrm{bps}$. UART transfers digitized EMG from click to microcontroller; because everything is happening in real time, we would have a problem using low speeds since the data simply would not be all sent. The lower bound for the transmission speed should be significantly above the top for the frequency that our bumpers come from. If the sampling frequency was $10 \mathrm{kHz}$ - values would be taken on every $100 \mu \mathrm{s}$. If the UART speed is, for example, generally known 9600 bps - we see that $104.2 \mu$ s is needed to transfer one bit, i.e. $1042 \mu$ s for one byte. A/D converters of less than 8-bits are not used in these applications; PIC18F87K22 contains a 12-bit ADC. We get that for every sample, for every $100 \mu$ s, we need 12 bits, and we have only 8 bits available at every $1042 \mu$ s. So 8 bits need to be transferred for the maximum of $66 \mu$ s.

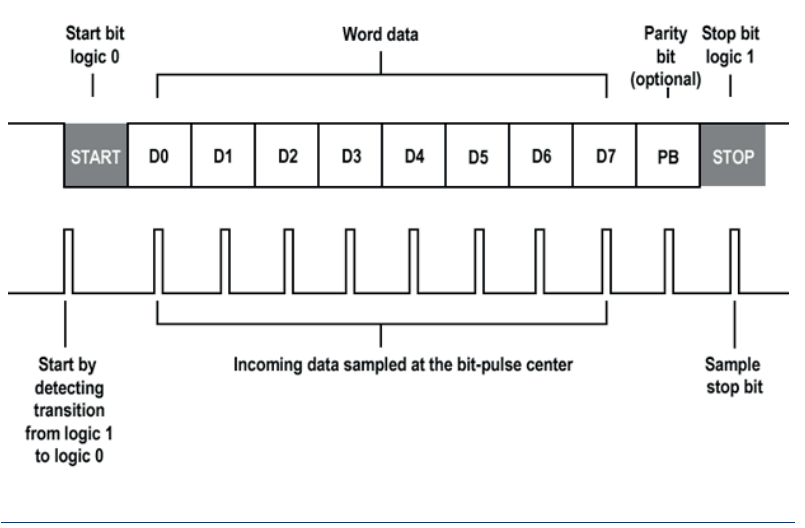

Fig. 4. UART code word

$$
\text { Baud rate }=8 \times(1 \div 0.000066)=121212.1 \text { bps }
$$

The first higher value defined with UART standard should be chosen.

\section{Click-board}

The EMG click serves for single-channel collection of electrical signals from motor neurons of skeletal muscles. It is placed on one of the microcontrollers mikroBUS sockets, and uses six of the sixteen possible pins (see Fig. 5) - two are for the mass and UART, and one for the power supply (5 V) and an analog EMG signal.

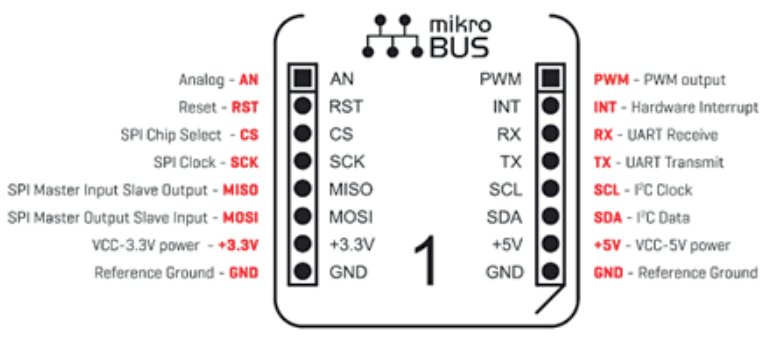

Fig. 5. MikroBUS standard

Before digitization, analog signal must be amplified and filtered - electronic circuits with operational amplifiers are used for that purpose. The electrode signal is run to, from first to last: preamplifier (instrumentation amplifier), HP filter, amplifier, HP filter, LP filter, AN pin of the microbus. All pins are located within the MCP609 amplifier chip. HP filters are passive and first order, and used because it would be technologically more costly to make one to equally weaken the signal. The LP filter is of the third order - the second row active is serial connected to the first order passive.

The DRL circuit takes a signal from the input of the preamplifier and takes it through two inverters, the other being the HP filter. Two-way zener diode (against high voltage) and diode pairs (against electrostatic discharge) are used as protection for the measuring channel and the DRL electrode. Apart from the amplifier, the MAX6106 chip is also used to form a reference mass for the entire circuit; in this case it is 2,048 V. This moves the base line of the EMG signal up from zero - otherwise negative values would be cut off. In case range of enhanced EMG needs to be adjusted to another type of microcontroller, with a different input range of A/D converter, a jumper and potentiometer (within MAX6106) move the reference mass. 


\section{FIRMWARE AND SOFTWARE}

MikroC is a software environment for managing PIC microcontrollers with $\mathrm{C}$ programming language. The user has at his disposal (among other things) a numbertype converter, several structures for hierarchical navigation within the project, an expandable library with a note on the applied functions and a helpful algorithm for clarifying the errors. The essence of the code (the algorithm for solving this particular problem) is written within the main function "void main ()", and other functions are defined out of it, but called within. Common example for that would be the interrupt routine. The body of the function is defined with curly brackets, and the variables that do not belong to each are automatically global. Fig. 6 shows the entire code needed to activate the necessary components in our system for acquiring and transferring bioelectric signals. For some parts of the code, the theoretical knowledge is necessary

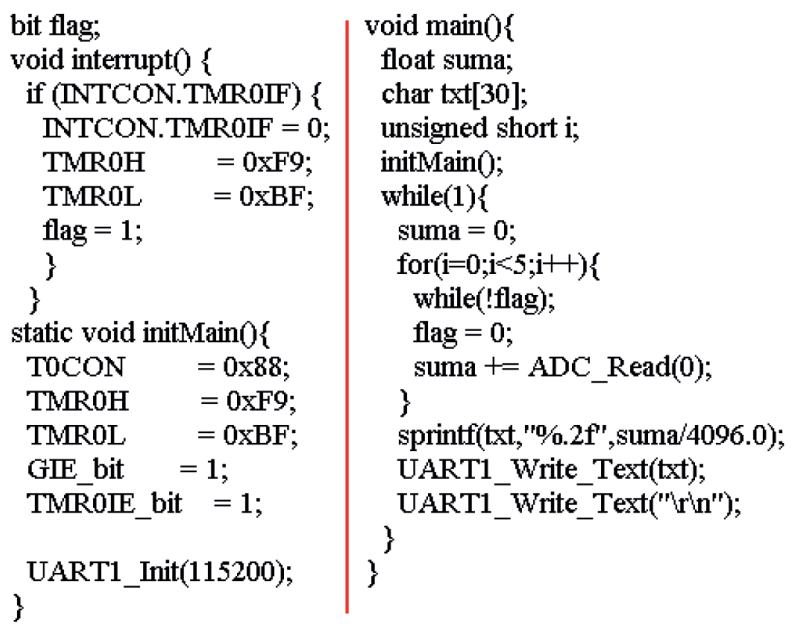

Fig. 6. Complete mikroC code

- Since the EMG frequency up to $500 \mathrm{~Hz}$ is considered, the Nyquist criterion states that the signal should be sampled with at least $2500 \mathrm{~Hz}$. Based on this and the logic shown in formula (3), a Baud rate greater than 30303 bps is required (we used 115200).

- For $2500 \mathrm{~Hz}$ gives a time interval of $0.4 \mathrm{~ms}$. PICs timer is incremented at every $0.25 \mu$ s - divide the time interval with it and it's shown that 1600 increments are needed in order to say with certainty that $0.4 \mathrm{~ms}$ have passed. Conclusion: values must be taken after every 1600 countdown - i.e. interrupt must appear.
- The timer register has 16 bits; it counts from 0 to 65535 - in the end an interrupt will automatically occur - it is a hypothetical bit that is out of the counting frame (modeled with a flag), when the registry itself is set to 16 zeros. Therefore, we introduce that every counting cycle starts 1600 increments before the end - hexadecimal variables TMROH and TMROL tell us that counting ranges from 63935.

- Assuming that the noise has Gaussian distribution; mentioned in [4], it can be filtered by arithmetic mean. Therefore, when calling an interrupt, a loop is given - every five consecutive values will be averaged. Five are taken for one more reason: when reading the values from the $\mathrm{A} / \mathrm{D}$ converter we get them in the voltage quants, and they need to be displayed in volts. The conversion is done by multiplying with $5 \mathrm{~V}$ (supply voltage) and dividing by $4096\left(2^{12}\right)$; two fives cancel each other out. Later, when the signal is being processed on a computer, the time axis must be multiplied by 5 , in order to be scaled properly.

\section{Signal display}

UART is compatible with many third-party programs for recording registered values. The files obtained in this way are in the .log format that can be entered in a spreadsheet like Microsoft Excel. From there they can be loaded into MATLAB. Fig. 7 shows us an example of EMG signal.

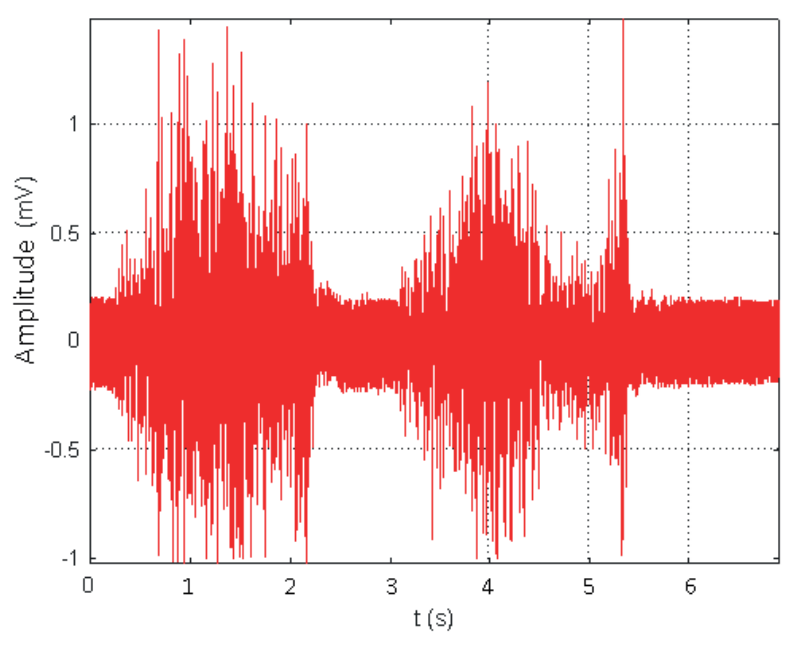

Fig. 7. EMG signal 
The respondent performed three rapid biceps contractions; without complete relaxation between the second and third one. Because the muscles are tightened even in a state of complete rest, values will exist at all times. Due to the elevation of the base signal line, a oneway component is hidden within the EMG - it was removed by subtracting its mathematical approximation from the entire signal. Also, $1 \%$ of the highest values in the signal were taken as a result of the pulse noise, and were deleted by direct and inverse vector sorting.

\section{REFERENCES}

[1] M.B. Reaz, M.S. Hussain, F. Mohd-Yasin. (Mar. 2006). Techniques of EMG signal analysis: detection, processing, classification and applications. Presented at Biological Procedures Online [Online]. Available: https://biologicalproceduresonline. biomedcentral.com/track/pdf/10.1251/bpo115

[2] Day, Scott. (2002). Important factors in surface EMG measurement. Bortec Biomedical Ltd Publishers. 1-17.

[3] D. Popović, M. Popović, M. Janković, Biomedicinska merenja i instrumentacija, Akademska misao, Belgrade, 2010.

[4] A. Phinyomark, C. Limsakul and P. Phukpattaranont, "EMG Feature Extraction for Tolerance of White Gaussian Noise," in Proc. Int. Work. Symp. Science and Technology (I-SEEC 08), Nong Hai, Thailand, Dec. 2008, pp. 178-183. 